\title{
弱环境辐射下中短波双波段红外图像融合方法
}

\author{
张润琦 ${ }^{1}$, 杨 波 $^{1}$, 夏丽昆 ${ }^{2}$, 赵灿兵 ${ }^{1}$, 苏俊波 ${ }^{1}$
}

(1. 昆明物理研究所, 云南 昆明 650223; 2. 陆军装备部驻重庆地区军事代表局驻昆明地区第一军事代表室, 云南 昆明 650032)

\begin{abstract}
摘要: 本文介绍了一种用于弱环境辐射下红外中、短波双波段的红外图像融合方法。该方法先采用单 尺度 Retinex（Single Scale Retinex, SSR）方法处理短波图像，分别对处理后的短波图像和中波图像 采用均值滤波和双边滤波得到细节层和背景层，再利用基于 Spectral Residual（SR）显著性和引导滤 波的权重映射, 对短波图像和中波图像的细节层和背景层进行加权融合, 得到融合图像。经过实验仿 真，并与基于小波、拉普拉斯金字塔的多种算法做了比较，该方法在各场景下的主观与客观评价均表 现良好。
\end{abstract}

关键词: 中短波红外, 图像融合, 双波段, 弱环境辐射, 引导滤波

中图分类号：TP391.41 文献标识码：A 文章编号：1001-8891(2020)05-0440-07

\section{Infrared Image Fusion Method for Medium-wave and Short-wave Images in Weak Ambient Radiation}

\author{
ZHANG Runqi ${ }^{1}$, YANG Bo ${ }^{1}$, XIA Likun ${ }^{2}$, ZHAO Canbing ${ }^{1}$, SU Junbo ${ }^{1}$ \\ (1. Kunming Institute of Physics, Kunming 650223, China;
}

2. The first military representative office in Kunming of the army armament department in Chongqing, Kunming 650032, China)

\begin{abstract}
This study introduces a method of infrared image fusion for the infrared medium- and short-wave dual band. This method uses the single scale retinex(SSR) method for processing the short-wave image. Following this, the short-wave and medium-wave images are processed to obtain the details and background layers by average filtering and bilateral filtering, respectively. Using the weight map based on spectral residual significant and guided filter, the background and details layers of the short-wave and medium-wave images are fused to obtain the final fused image. From the results of the experimental simulation and a comparison with various fusion algorithms based on wavelet and the Laplacian pyramid, the method proposed in this work is observed to be simple and performs well in most scenarios.
\end{abstract}

Key words: medium and short wave infrared image, image fusion, dual band, low environmental radiation, guided filter

\section{0 引言}

随着科技的不断发展，各国对红外探测成像技术 的要求也越来越高。由于传统单波段红外成像技术难 以解决复杂背景下真伪目标识别等难题, 双波段及多 波段红外技术成了未来的发展方向。其中融合算法是 多波段成像技术中极为重要的一部分, 在这一领域, 国内外学者已进行了大量的研究工作，包括像素级、 特征级及决策级融合算法。像素级融合主要方法有基 于金字塔变变换、小波变换和神经网络方法等, 特征 级融合算法包括基于聚类分析、信息熵的方法等, 决 策级融合算法包括基于神经网络、贝叶斯估计等的方
法 ${ }^{[1]}$ 。

这些方法有效但需要针对应用场景加以改进, 在 制定融合策略时需要根据波段特性进行设计。相对于 中长波红外融合领域, 中短波融合研究较少, 且短波 红外与中、长波有很大的成像效果差异。短波红外的 波长范围为 $0.75 \sim 3 \mu \mathrm{m}$, 其主要利用环境中的辐射成 像, 而自然环境中的辐射源主要为太阳辐射及反射, 成像效果类似于可见光图像, 拥有更多的纹理细节, 但对比度在环境辐射弱的时候较低。中波红外的波长 范围为 3 $5 \mu \mathrm{m}$, 其主要靠物体自身辐射成像, 在湿 热环境下探测能力更强, 热目标更明显 ${ }^{[2]}$ 。图 1 展示 了中短波双波段探测器在下午 3:00 采集到的一组图 
片, 此时自然环境中辐射较强, 可以看出短波图像拥 有更多的纹理细节, 图片内包含的更多是太阳辐射反 射带来的信息, 效果和可见光类似。中波图像缺少纹 理信息, 但对热目标更敏感。图 2 展示了同一台红外 探测器在傍晚 7:30 采集到的图像, 此时自然环境中辐 射非常弱, 短波图像细节信息较白天弱了很多, 对比 度也降低了很多, 而中波图像目标和背景轮廓更鲜 明。针对以上中短波特性, 本文提出了一种针对弱背 景辐射条件下的中短波双波段融合算法。

\section{1 算法分析}

根据人眼成像特点, Edwin Land 通过研究其逆过 程, 提出了 Retinex 理论, 并成功用于图像增强、去 雾等多个领域 ${ }^{[3]}$ 。对比传统直方图均衡的方法, 基于 Retinex 理论的方法克服了原始图像多个灰度级在处 理过程中合成一个灰度造成细节丢失的问题。Retinex 模型中, 将图像分解为亮度图像和反射图像, 通过改 变亮度图像和反射图像之间的比例来达到图像增强 的目的 ${ }^{[4]}$ 。基于 Retinex 理论的增强算法已在可见光 图像增强领域中广泛使用, 短波图像与可见光图像相 似，拥有更多的细节，但在外界辐射光源较微弱的时 候, 总体像素值普遍偏低, 呈现画面较暗对比度较低, 因此适合采用单尺度 Retinex 图像增强算法做融合前 的增强处理。灰度图 SSR 实现原理如公式(1)、(2)、 (3)所示:

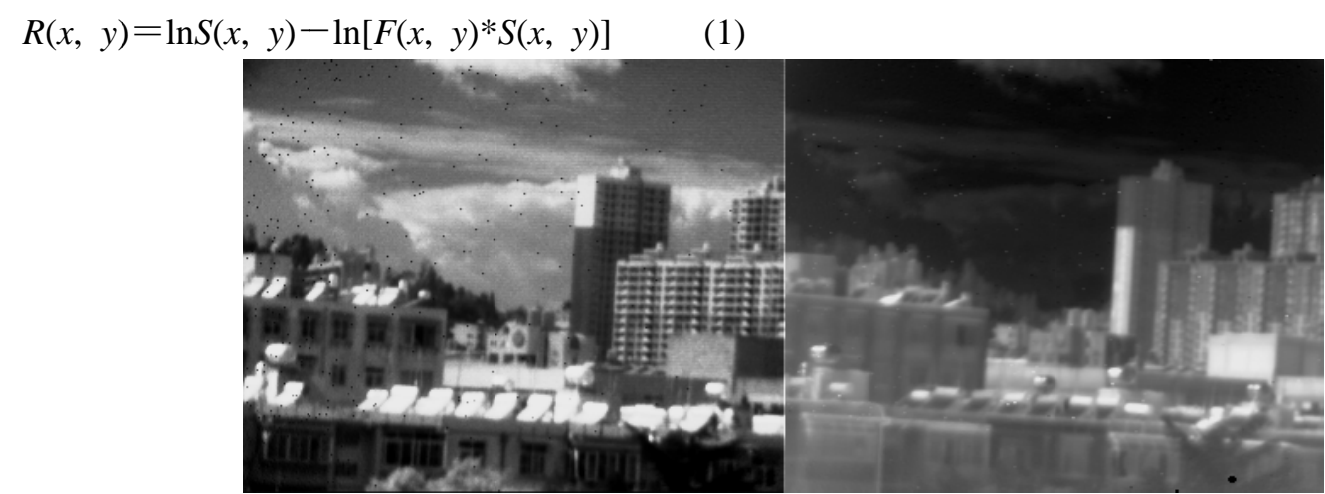

图 1 白天短波 (左) 和中波 (右) 图像对比

Fig.1 Short wave by daylight(left) and medium wave(right) contrast
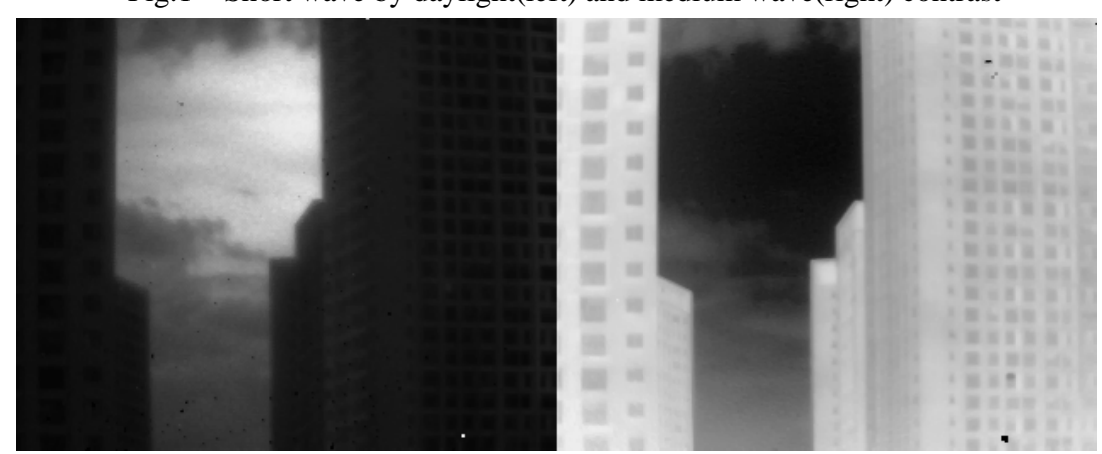

图 2 傍晚短波 (左) 和中波 (右) 图像对比

$$
\begin{gathered}
F(x, y)=K \times \exp \left[-\frac{x^{2}+y^{2}}{c^{2}}\right] \\
\iint F(x, y) \mathrm{d} x \mathrm{~d} y=1
\end{gathered}
$$

式中: $R(x, y)$ 为输出图像; $S(x, y)$ 为输入图像; $*$ 为卷 积运算; $F(x, y)$ 为环绕函数; $\ln$ 为自然对数运算; $C$ 为 高斯函数标准偏差; $K$ 的取值应满足公式(3)。

弱背景辐射下的短波图像经过 SSR 处理后如图 3 所示, 可以看到原图在经过 SSR 增强后, 图像对比度 有了较大改善, 左上角物体和右侧细节更为清晰地展 现在了人眼面前, 经过 SSR 增强后的短波图像更有利 于提取显著性特征以及高频细节信息。

分别对短波图像和中波图像滤波提取高频细节 图层和低频背景图层, 对短波图像采取均值滤波, 但 由于中波图像缺乏细节但具有更分明的轮廓信息, 因 此对中波图像采用双边滤波。如高斯滤波等传统滤波 器在滤波的同时会滤除为高频信号的图像边界, 因此 图像包括边界在内的整体都会模糊。双边滤波则同时 关注滤波窗口中心点与其他点之间的灰度相似程度, 同时也关注他们之间几何邻近度，因此双边滤波具有 良好的保边特性。双边滤波定义表达式如公式(4)、(5)、 (6)、 (7)所示:

$$
R_{\mathrm{BF}}(x)=\frac{1}{C_{\mathrm{d}, \mathrm{r}}} \sum_{y \in \Omega}\left[w_{\mathrm{d}}(x, y) w_{\mathrm{r}}(x, y) u(y)\right]
$$




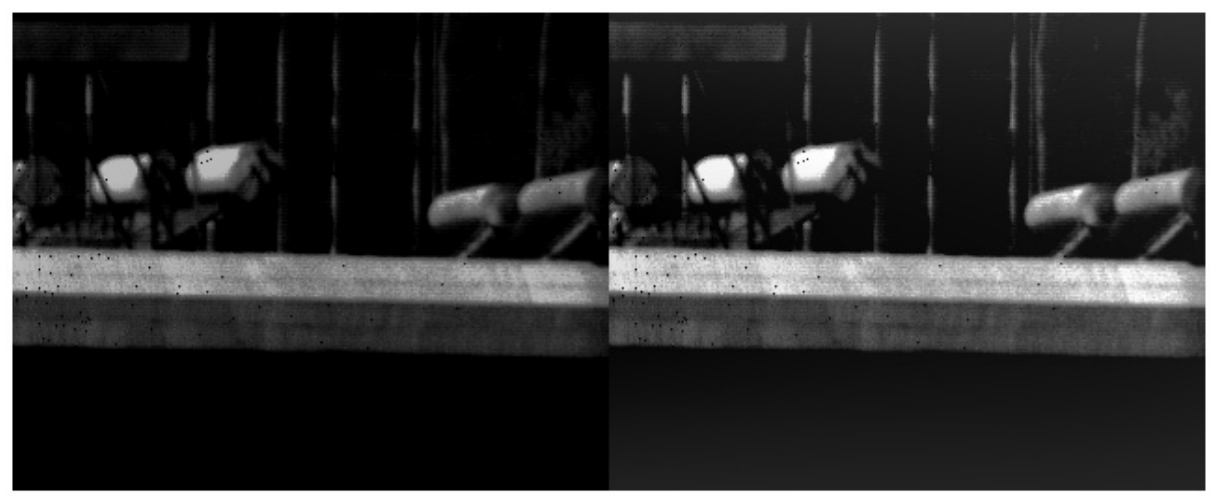

图 3 短波图像原图（左）与经过 SSR 增强后的图像（右）

Fig.3 Short wave image before (left) and after(right) SSR enhancement

式中:

$$
\begin{gathered}
w_{\mathrm{d}}(x, y)=\exp \left(-\frac{|x-y|^{2}}{2 \delta_{\mathrm{d}}^{2}}\right) \\
w_{\mathrm{r}}(x, y)=\exp \left(-\frac{|u(x)-u(y)|^{2}}{2 \delta_{\mathrm{r}}^{2}}\right) \\
C_{\mathrm{d}, \mathrm{r}}=\sum_{y \in \Omega}\left[w_{\mathrm{d}}(x, y) w_{\mathrm{r}}(x, y)\right]
\end{gathered}
$$

上面方程中, $x=\left(x_{1}, x_{2}\right)$ 为区域 $\Omega$ 中心像素点位置 坐标; $y=\left(y_{1}, y_{2}\right)$ 为区域 $\Omega$ 相邻像素点位置坐标; $R_{\mathrm{BF}}(x)$ 为输出图像; 权重 $w_{\mathrm{d}}(x, y)$ 和 $w_{\mathrm{r}}(x, y)$ 分别度量了区域 $\Omega$ 中心像素点 $x$ 与邻近像素点 $y$ 的几何邻近度与灰度相 似度; $\delta_{\mathrm{d}}$ 和 $\delta_{\mathrm{r}}$ 分别为空间距离权系数参数和图像灰度 权系数, $C_{\mathrm{d}, \mathrm{r}}$ 为归一化系数 ${ }^{[6]}$ 。

对中波原图和经过 SSR 增强后的短波图像进行 显著性分析, 本文采用的显著性计算模型是由上海交 大侯晓迪在 CVPR 上提出的 SR 模型 ${ }^{[7]}$, 该模型核心 思想如下: 人们的视觉对信号的变化部分更为敏感, 视觉系统抑制频繁出现的特征, 但对非常规的特征保 持敏感, 图像可分解为如公式(8)所示两个部分。

$$
H(\text { 原图 })=H(\text { 非常规变化 })+H(\text { 常规变化 })
$$

侯晓迪 ${ }^{[7]}$ 发现在图像样本非常大的时候, 经过傅 里叶变换后的振幅谱取对数平均值正比于它们的频 率, 因此单独一幅图像的振幅谱取对数后减去大量图 像样本平均振幅谱取对数的值即为该图像的显著性 部分。

对获得的两幅显著性图像每个像素点都取两个 矩阵中大的值, 就得到了一幅显著值图, 再用这两个 图分别和这个显著值图去比较, 如果某点的像素值相 等, 那显著权重图就是 1 , 否则就是 0 , 得到二值显 著权值图像。使用二值显著权值作为引导图像, 分别 对中波原图和经过 SSR 增强的短波图像进行引导滤
波得到两个波段的权重图。He 等人在 2010 年首次提 出了引导滤波 (Guide Filter) 算法 ${ }^{[8]}, 2013$ 年 $\mathrm{Li}$ 等人 也将基于显著性的引导滤波用于图像融合 ${ }^{[9]}$ 。引导滤 波将滤波过程当作一个加权和过程, 可由公式(9)表 示:

$$
R_{i}=\sum_{j} W_{i, j}(I) S_{j}
$$

式中: $R$ 为输出图像; $I$ 为引导图像; $S$ 为输入图像。

将引导图像和输出图像用一个线性模型表示, 如 公式(10)所示:

$$
R_{i}=a_{k} I_{i}+b_{k}, \quad \forall i \in w_{k}
$$

式中: $w_{k}$ 表示以像素点 $k$ 为中心, 大小为 $(2 r+1) \times(2 r$ $+1)$ 的窗口。 $a, b$ 的值由公式(11) 所示的代价函数 $E\left(a_{k}\right.$, $b_{k}$ ) 进行窗口 $w_{k}$ 最小化所确定, 由公式(11)可以解出公 式(12)和公式(13):

$$
\begin{gathered}
E\left(a_{k}, b_{k}\right)=\sum_{i \in w_{k}}\left[\left(a_{k} I_{i}+b_{k}-S_{i}\right)^{2}+\varepsilon a_{k}^{2}\right] \\
a_{k}=\frac{1}{\left(\delta^{2}+\varepsilon\right)|w|}\left(\sum_{i \in w_{k}} I_{i} S_{i}-\mu_{k} \overline{S_{k}}\right) \\
b_{k}=\overline{S_{k}}-a_{k} \mu_{k}
\end{gathered}
$$

式中: $|w|$ 为窗口内的像素个数; $\mu_{k}$ 和 $\delta^{2}$ 为输入图像在 窗口内的均值和方差; $\varepsilon$ 为平滑因子; $P$ 为滤波输入图 像 ${ }^{[10-11]}$

使用二值显著图作为引导图像引导滤波得到的 权重图对低频背景图层和高频细节层加权和, 得到最 终融合图像。该算法总体流程框架如图 4 所示。

\section{2 仿真及结果分析}

本文采用 $320 \times 256$ 中短波双波段红外探测器, 采集到了 4 组较为典型的场景图片进行仿真展示, 场 景一为下午 3:00 阳光充足背景辐射较强时所拍摄的 
天空与建筑图片, 此时短波图像细节丰富, 天空云层 次分明。场景二为与场景一相近场景于晚上 8:30 所拍 摄到的图片, 此时太阳完全落山, 环境辐射极弱, 由 于短波成像特点, 此时短波图像对比度极低, 细节损 失极大。场景三为下午 6:30 所拍摄到的建筑群及屋顶 的太阳能板和太阳能热水器, 此时太阳已快落山, 环 境辐射较白天弱, 可以看到中波和短波由于成像机理 差异, 太阳能板和太阳能热水器上所呈现的像素值截 然相反。场景 4 同样为晚上 8:30 所拍摄的照片, 短波 图像基本失去清晰成像能力。
作为对比所使用的融合算法包括小波融合算法 (Wavelet Fusion), 基于 Retinex 的拉普拉斯金字塔融合

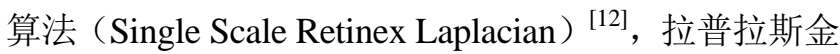
字塔（Laplacian Pyramid）融合方法和基于五株采样的 提升小波融合法 (Lifting Scheme Quincunx) ${ }^{[13]}$, 分别 简称为 “WF”、“SSRL”、“LELP”与 “LISQ”。上述 算法与本文提出算法融合图像在 4 个场景下效果对比 如图 5, 图 6, 图 7 和图 8 所示。

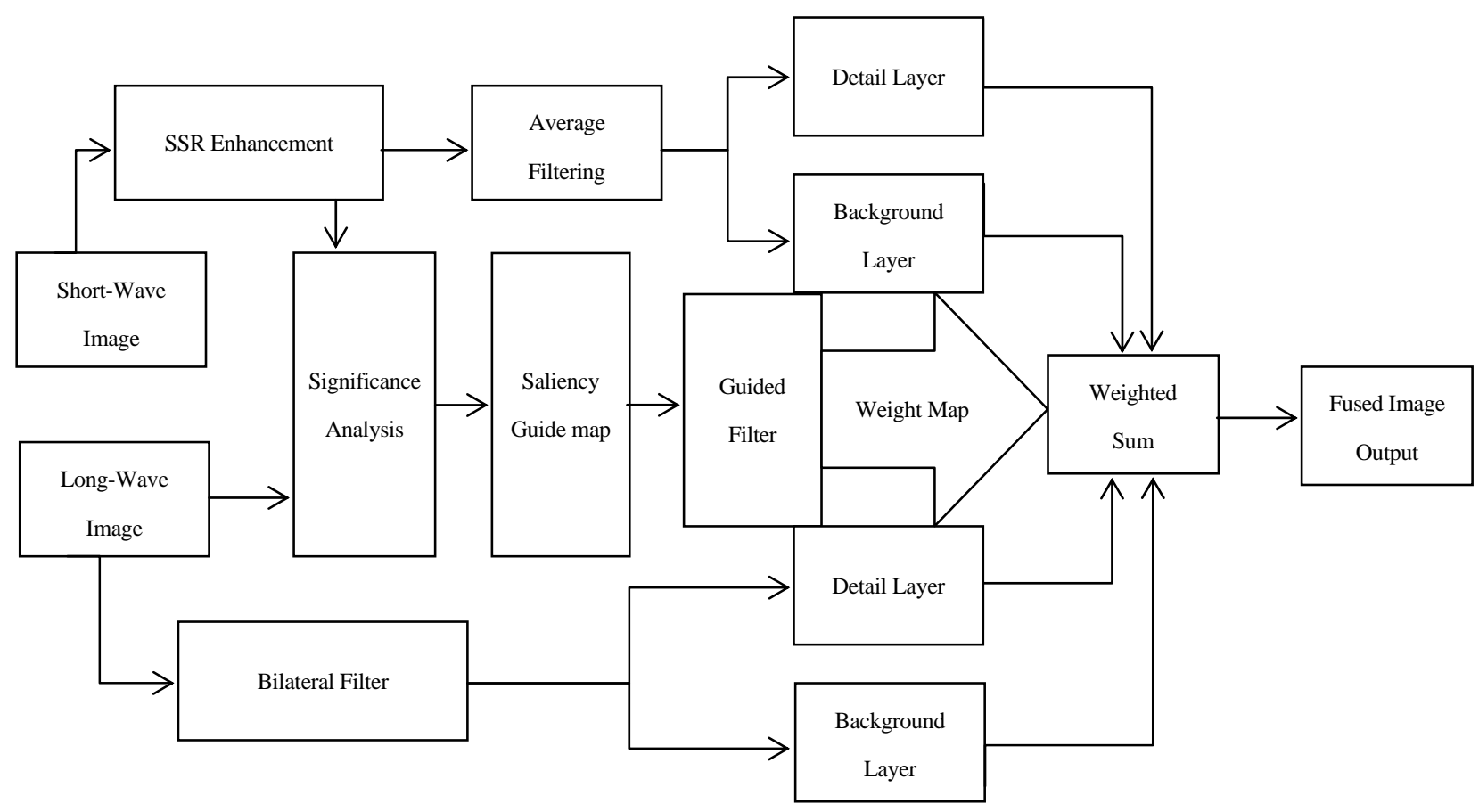

图 4 本文算法总体流程框架

Fig.4 The overall process framework of the algorithm in this paper

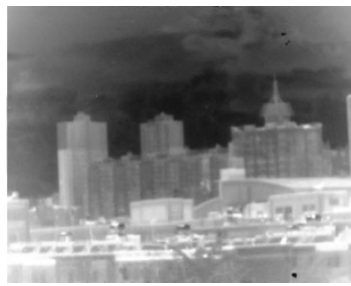

(a) Medium wave image

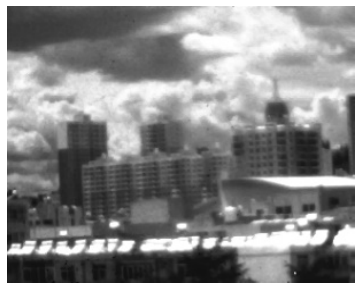

(b) Short wave image

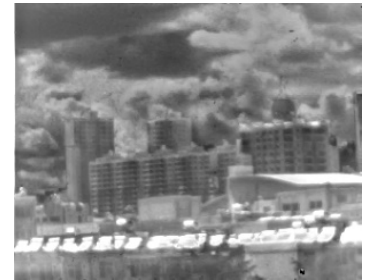

(c) SSRL fusion image

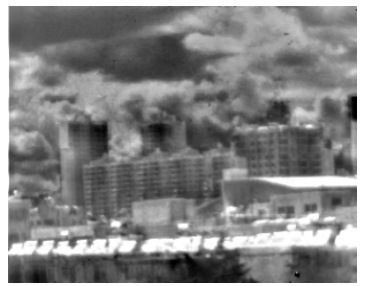

(d) LP fusion image

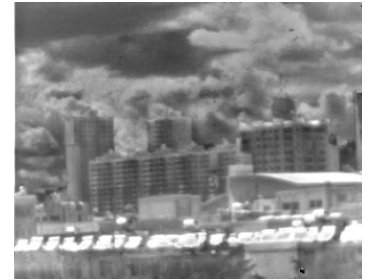

(e) LISQ fusion image

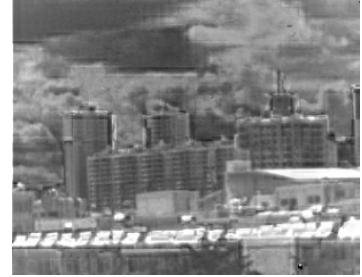

(f) WF fusion image

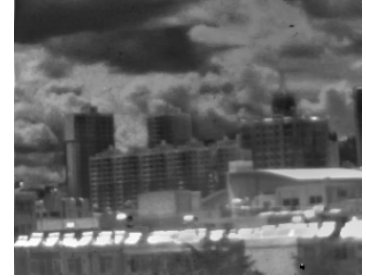

(g) 本文算法 (g) Algorithm in this paper

图 5 场景一融合效果对比

Fig.5 Scene 1 fusion effect comparison 


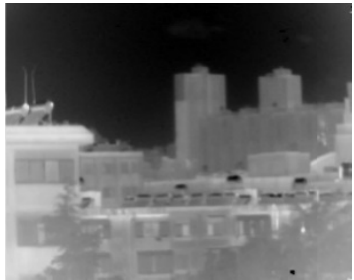

(a) Medium wave image

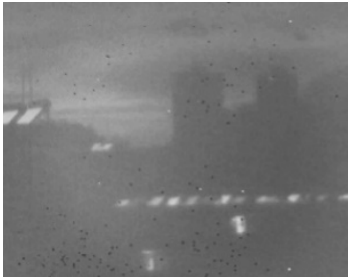

(b) Short wave image

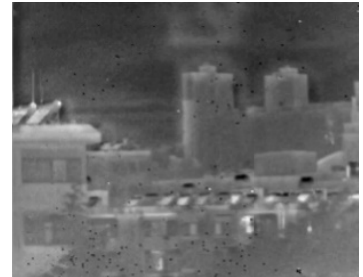

(c) SSRL fusion image

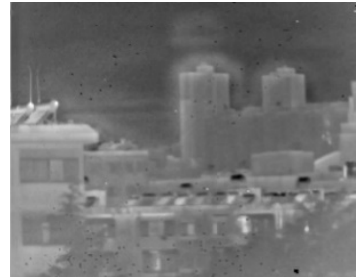

(d) LP fusion image

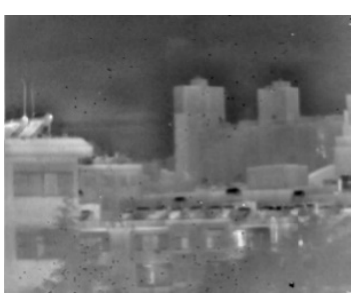

(e) LISQ fusion image

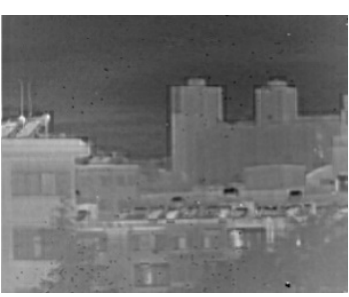

(f) WF fusion image

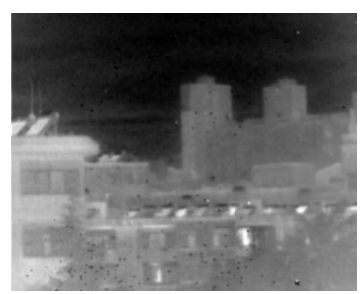

(g) Algorithm in this paper

图 6 场景二融合效果 Fig.6 Scene 2 fusion effect comparison

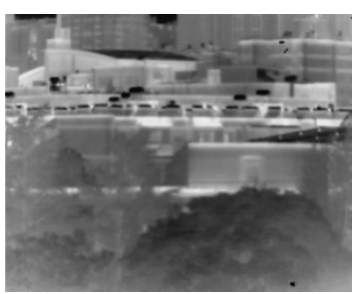

(a) Medium wave image

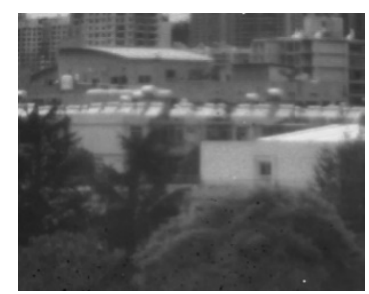

(b) Short wave image

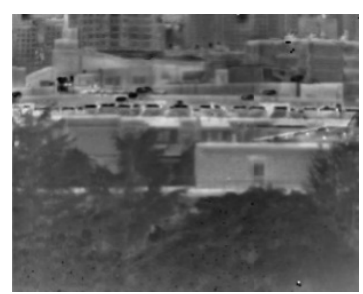

(c) SSRL fusion image

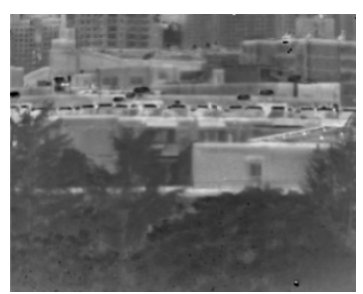

(d) LP fusion image

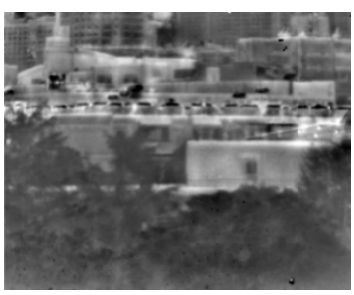

(e) LISQ fusion image

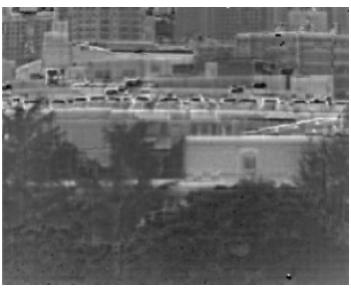

(f) WF fusion image

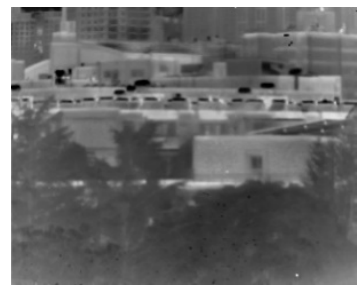

(g) Algorithm in this paper

图 7 场景三融合效果 Fig.7 Scene 3 fusion effect comparison

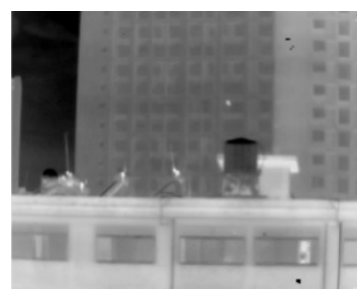

(a) Medium wave image

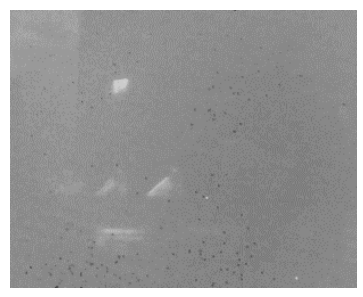

(b) Short wave image

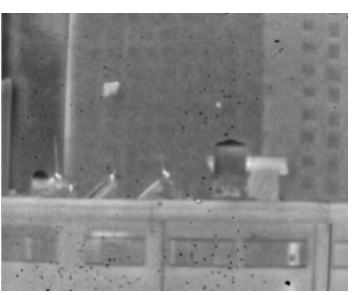

(c) SSRL fusion image

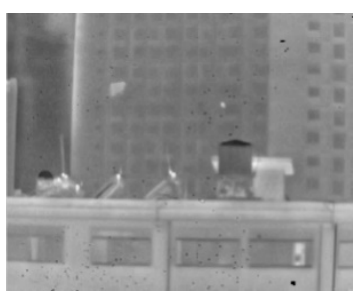

(d) LP fusion image

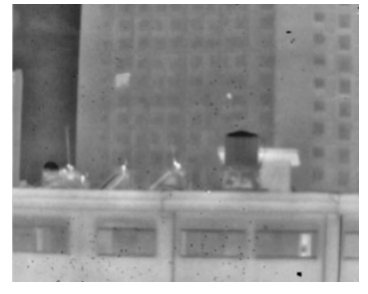

(e) LISQ fusion image

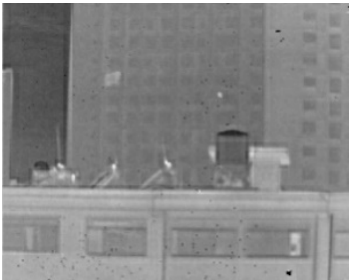

(f) WF fusion image

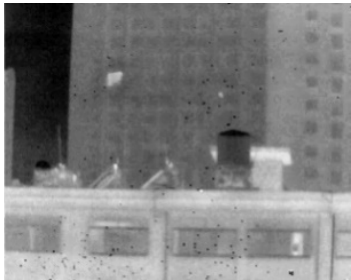

(g) Algorithm in this paper

图 8 场景四融合效果 Fig.8 Scene 4 fusion effect comparison 
从上面图像观感对比可以看出, 相较于其他算 法, 本文算法处理得到的融合图像细节丰富, 对比度 较大, 且图像灰度过渡均匀。在场景一中, 可以看出 在白天阳光充足背景辐射强时, 各算法表现均良好, 由于本文算法短波图像经过 SSR 增强处理, 在对比度 和细节上表现更好一些。但在场景二中, 此时环境辐 射弱, 短波图像只有少部分太阳能热水器的信息, 除 了本文算法和传统小波算法, 其他融合均出现了因融 合灰度巨大反差形成的灰度过渡不均匀现象, 但传统 小波融合由于其本身融合机理, 呈现块效应。本文融 合算法则很好地保留了中波图像的边界轮廓信息, 图 像看上去更加自然。场景三和场景四本文算法相比其 他算法轮廓更清晰, 对比度更大, 图像更均匀, 场景 与目标过渡也更自然。

目前为止, 没有一种可以全方位评价融合图像质 量的客观指标, 且单一指标也无法适用于所有目标场 景。因此下面使用了 7 个常用的图像融合的客观评价 指标来评价上面 5 种算法好坏, 包括相对标准差 (Relative Standard Deviation)、信息熵（Entropy）、均
方根误差 (Mean-root-square Error)、峰值信噪比 (Peak Signal-to-noise Ratio)、互信息 (Mutual Information)、 结构相似性（Structural Similarity）和交叉熵（Cross Entropy), 分别记为“RSTD”、“IE”、“MSE”、“PSNR”、 “MI”、“SSIM” 和 “CE”。相对标准差与均方误差反 映了融合图像与源图像灰度相似程度, 评价值越小, 说明融合图像越相似。信息熵用于衡量融合图像中信 息丰富程度, 评价值越大说明融合图像信息越丰富。 峰值信噪比用于衡量有效信息与噪声之间的比率, 评 价值越大说明图像有效信息更多。结构相似度衡量融 合图像与源图像的相似性, 评价值越大说明融合图像 亮度、对比度和结构越相似, 融合质量越好。交叉熵 反映了融合图像与源图像所含信息量的相对衡量, 评 价值越大, 融合图像与源图像相似性越大。互信息度 量了融合图像保留了源图像信息量的多少, 评价值越 大说明融合效果越好 ${ }^{[14]}$ 。4 个场景各个融合算法的上 述 7 个客观评价指标统计如表 1 , 表 2, 表 3 和表 4 所示。

\begin{tabular}{|c|c|c|c|c|c|c|c|}
\hline & RSTD & IE & MSE & PSNR & MI & SSIM & CE \\
\hline LP & 0.6983 & 7.0533 & 0.0378 & 143.5772 & 1.0575 & 0.6359 & 0.00046 \\
\hline LISQ & 0.4230 & 7.3514 & 0.0360 & 144.0484 & 1.0659 & 0.6219 & 0.00321 \\
\hline SSRL & 0.6983 & 7.0533 & 0.0378 & 143.5772 & 1.0575 & 0.6359 & 0.00046 \\
\hline WF & 0.7120 & 7.0819 & 0.0369 & 143.8066 & 1.1056 & 0.5999 & 0.00042 \\
\hline The proposed algorithm & 0.6715 & 7.1877 & 0.0278 & 146.6491 & 1.1960 & 0.6375 & 0.00042 \\
\hline
\end{tabular}

表 2 场景二各评价指标 Table 2 Scene 2 each evaluation index

\begin{tabular}{llllllll}
\hline & RSTD & IE & MSE & PSNR & MI & SSIM & CE \\
\hline LP & 0.9778 & 6.7029 & 0.0360 & 144.0798 & 2.3818 & 0.7063 & 0.00400 \\
LISQ & 1.1419 & 6.9642 & 0.0352 & 144.2821 & 2.4221 & 0.7036 & 0.00267 \\
SSRL & 1.0888 & 6.8608 & 0.0309 & 145.5929 & 2.3199 & 0.6684 & 0.00244 \\
WF & 1.0305 & 6.7908 & 0.0350 & 144.3418 & 2.7750 & 0.6886 & 0.00451 \\
The proposed algorithm & 2.0165 & 7.4982 & 0.0272 & 146.8759 & 3.9485 & 0.6861 & 0.00313 \\
\hline
\end{tabular}

表 3 场景三各评价指标 Table 3 Scene 3 each evaluation index

\begin{tabular}{llllllll}
\hline & RSTD & IE & MSE & PSNR & MI & SSIM & CE \\
\hline LP & 0.2042 & 6.9546 & 0.0324 & 145.1092 & 1.7012 & 0.6801 & 0.00071 \\
LISQ & 0.0504 & 7.1408 & 0.0318 & 145.2990 & 1.4055 & 0.6514 & 0.00314 \\
SSRL & 0.3161 & 6.9262 & 0.0269 & 146.9812 & 1.7675 & 0.6610 & 0.00056 \\
WF & 0.2732 & 6.9109 & 0.0324 & 145.1070 & 1.5294 & 0.6413 & 0.00074 \\
The proposed algorithm & 0.3704 & 6.9180 & 0.0274 & 146.7777 & 2.4039 & 0.6889 & 0.00356 \\
\hline
\end{tabular}


表 4 场景四各评价指标

Table 4 Scene 4 each evaluation index

\begin{tabular}{llllllll}
\hline & RSTD & IE & MSE & PSNR & MI & SSIM & CE \\
\hline LP & 2.3660 & 6.5891 & 0.0340 & 144.6306 & 2.7049 & 0.7759 & 0.01617 \\
LISQ & 2.9775 & 6.8790 & 0.0330 & 144.9534 & 2.5922 & 0.7766 & 0.01739 \\
SSRL & 2.4997 & 6.6864 & 0.0272 & 146.8823 & 2.2884 & 0.7072 & 0.01617 \\
WF & 2.4573 & 6.6157 & 0.0334 & 144.8294 & 3.0017 & 0.7554 & 0.01739 \\
The proposed algorithm & 4.7734 & 7.416 & 0.0227 & 148.6819 & 4.2383 & 0.7210 & 0.00516 \\
\hline
\end{tabular}

可以看出, 在以上所拍摄的试验场景中, 本文融 合算法的信息熵、结构相似度、互信息、均方误差、 峰值信噪比几个指标较其他算法有明显优势, 说明本 文算法更好地获取了两个波段的细节, 尤其在弱环境 辐射背景下比其他算法有着更多的信息。

\section{3 结论}

随着当今成像技术向着双波段甚至多波段、多光 谱趋势发展, 多种双波段及多波段的融合算法也相继 被提出, 但这些算法针对弱环境辐射下的红外中短波 双波段较少。本文基于红外中短波两个波段的图像特 点, 提出了一种适用于弱环境辐射的图像融合方法, 该方法原理简单, 并使用中短波碲镉录红外探测器实 际采图，与其他算法做了对比试验。实验结果表明， 该方法较好地保留了两个波段各自的显著性特点, 增 强了融合图像中的短波细节，扩大了整体对比度，保 留了中波图像轮廓信息, 且不会产生基于拉普拉斯相 关融合算法在有些场景出现融合效果不自然的现象。

\section{参考文献:}

[1] 李朝阳. 红外多波段图像融合算法研究[D]. 成都: 中国科学院大学 (中国科学院光电技术研究所), 2018

LI Zhaoyang. Research on multi band infrared image fusion algorithm[D]. Chengdu: University of Chinese Academy of Sciences(Institute of Optics and Electronics, Chinese Academy of Sciences), 2018.

[2] 朱小红. 多波段红外图像差异特征形成机理研究[D]. 太原: 中北大 学, 2016.

ZHU Xiaohong. Research on the forming mechanism of multi-band infrared images'difference features[D]. Taiyuan: North University Of China, 2018.

[3] Jobson D J, Rahman Z, Woodell G A. Properties and performance of acenter/surround retinex[C]//Image Processing, IEEE, 2002, 6(3): 451-462.

[4] Meylan L , Süsstrunk, Sabine. Color Image Enhancement Using A Retinex-Based Adaptive Filter[C]//Conference on Colour in Graphics, 2004: 359-363
[5] 付国文. 基于 Retinex 的图像增强算法研究及实现[D]. 上海: 上海交 通大学.

FU Guowen. Research of Image Enhancement Algorithms Based On Retinex And Implementation[D]. Shanghai: Shanghai Jiao Tong University.

[6] 蒋辉. 双边滤波理论及其在遥感图像处理中的应用研究 [D]. 成都: 西 南交通大学, 2014

JIANG Hui. Research For Bilateral Filtering Theory And ItsApplication In Remote Sensing Image Processing[D]. Chengdu: Southwest JiaoTong University, 2014

[7] HOU Xiaodi, ZHANG Liqing. Saliency Detection: A Spectral Residua Approach[C]//Computer Vision and Pattern Recognition, IEEE, 2007, 17(27): 1063-6919.

[8] HE K, SUN J, TANG X. Guided Image Filtering[M]. Berlin: Computer Vision-ECCV Springer Berlin Heidelberg, 2010.

[9] LI S, KANG X, HU J. Image Fusion With Guided Filtering[C]//IEEE Transactions on Image Processing, 2013, 22(7): 2864-2875.

[10] 姚吉. 基于引导滤波的 NSCT 域图像融合方法研究[D]. 南昌: 南昌航 空大学, 2018 .

YAO Ji. Research on Image Fusion Based on Guide Filter in NSCT domain[D]. Nanchang: Nanchang Hangkong University, 2018.

[11] 李甜甜. 基于导向滤波和多尺度分析的图像融合算法研究[D]. 西安: 西安电子科技大学, 2015.

LI Tiantian. Image fusion based on Guided filter and Multi-scale analysis[D]. Xi’an: Xidian University, 2015.

[12] WU R, YU D, WU H, et al. An Improve Fusion Method For Infrared And Low-Light Level Visble Image [R]. ICCWAMTIP, 2017.8301467

[13] 祝青, 刘斌. 提升五株形小波在多光谱图像融合中的应用[J]. 计算机 工程与应用, 2012, 48(11): 170-177.

ZHU Qing, LIU Bin. Application of lifting quincunx in multispectral image fusion[J]. Computer Engineer and Applications, 2012, 48(11): 170-177.

[14] 张小利, 李雄飞, 李军. 融合图像质量评价指标的相关性分析及性能 评估[J]. 自动化学报, 2014, 40(2): 306-315.

ZHANG Xiaoli, LI Xiongfei, LI Jun. Validation and Correlation Analysis of Metrics for Evaluating Performance of Image Fusion[J]. Acta Automatica Sinica, 2014, 40(2): 306-315. 